\title{
The Clinical Indications and Cytogenetic Analysis of Genetic
} Amniocentesis Cases

\author{
Seyed Mehdi Tabatabaei (iD ${ }^{1,}{ }^{*}$ and Zahra Metanat ${ }^{2}$ \\ ${ }^{1}$ Pregnancy Health Research Center, Zahedan University of Medical Sciences, Zahedan, Iran \\ ${ }^{2}$ Provincial Clinical Genetic Counseling Center, Ali-Asgahr Pediatric Hospital, Zahedan, Iran \\ "Corresponding author: Pregnancy Health Research Center, Zahedan University of Medical Sciences, Zahedan, Iran. Email: zu.healthdeputy@gmail.com \\ Received 2019 January 24; Revised 2019 March 05; Accepted 2019 July 14.
}

Keywords: Prenatal Screening, Genetic Amniocentesis, Genetic Counseling, Iran

\section{Dear Editor,}

Prenatal genetic screening tests are planned to screen for structural abnormalities, aneuploidy, and genetic diseases in the prenatal period. The prevalence of genetically modified disorders in neonates is estimated to be $4 \%$, and the risk increases with increasing maternal age (1). Down syndrome is one of the most prevalent genetic diseases affecting about 1 in 400 - 1500 newborns, and the risk increases with increasing maternal age (2). Screening for this syndrome is recommended to all pregnant women, especially in women 35 years of age and older.

Prenatal genetic screening tests of pregnant woman include first-trimester screening, second-trimester screening, and combined first- and second-trimester screening tests. First-trimester screening (FTS) for common chromosomal aneuploidies is based on a combination of maternal age and biochemical serum measurements of free beta-human chorionic gonadotropin (B-HCG), pregnancy-associated plasma protein-A (PAPP-A), and ultrasonographic evaluation of nuchal translucency (NT). These tests are usually performed between 11 weeks and 13 weeks of pregnancy. Second-trimester screening (STS) includes biochemical serum measurements of triplemarkers (alpha-fetoprotein, unconjugated estriol, and B-HCG) or quad-markers (alpha-fetoprotein, unconjugated estriol, B-HCG, and inhibin A), and ultrasound exams that screen the fetus for anomalies. The tests are usually done between 15 weeks and 22 weeks of pregnancy. However, in Iran, because of legal constraints for therapeutic abortions after the 20th week of pregnancy, STS is mostly recommended before the 17th week of pregnancy.

The detection rate of down syndrome for the firsttrimester screening at earlier stages of pregnancy is higher than the second-trimester screening (approximately 87\% versus $81 \%$ ) (3). The screening tests that involve two markers, specifically PAPP-A, free $\beta$-hCG, in combination with maternal age have a considerably better performance than those involving single markers with and without maternal age, and they can detect $70 \%$ of down syndrome affected pregnancies (4). However, underutilization of the first-trimester screening shows that the advantage of earlier diagnosis associated with FTS has been overlooked by clinicians providing genetic counseling.

The risk of fetal loss and other complications associated with amniocentesis is very low, and the risks of invasive procedures have also decreased over time (5). However, not all women with abnormal screening test results agree to undertake such an invasive procedure. Offering pregnant women the relatively new noninvasive prenatal screening(NIPS) tests that use cell-free fetal DNA sequences isolated from a maternal blood sample may provide reassurance to women who have concerns about undertaking an amniocentesis (6).

GA is recommended for pregnant women with abnormal screening tests. FTS has acceptable performance for detection of pregnancies with chromosomal abnormalities; however, this method has been hardly recommended by clinicians. Some pregnant women who are GA candidates refuse invasive prenatal diagnosis. Moreover, a large fraction of pregnant women have a delayed diagnosis or recommendations for therapeutic abortion. Training of the clinicians on the principles of genetic counseling and increasing public awareness regarding prenatal genetic screening tests and providing pre- and post-test genetic counseling is of great importance. 


\section{Footnotes}

Conflict of Interests: The authors declare that there is no conflict of interests regarding the publication of this paper.

Funding/Support: This study was supported by Zahedan University of Medical Sciences.

\section{References}

1. Wieacker P, Steinhard J. The prenatal diagnosis of genetic diseases. Dtsch Arztebl Int. 2010;107(48):857-62. doi: 10.3238/arztebl.2010.0857. [PubMed: 21173933]. [PubMed Central: PMC3004373].

2. Asim A, Kumar A, Muthuswamy S, Jain S, Agarwal S. "Down syndrome: An insight of the disease".J Biomed Sci. 2015;22:41. doi: 10.1186/s12929015-0138-y. [PubMed: 26062604]. [PubMed Central: PMC4464633].
3. Seyyed Kavoosi E, Younessi S, Farhud DD. Screening of fetal chromosome aneuploidies in the first and second trimester of 125,170 Iranian pregnant women. Iran J Public Health. 2015;44(6):791-6. [PubMed: 26258091]. [PubMed Central: PMC4524303].

4. Alldred SK, Takwoingi Y, Guo B, Pennant M, Deeks JJ, Neilson JP, et al. First trimester serum tests for Down's syndrome screening. Cochrane Database Syst Rev. 2015;(11). CD011975. doi: 10.1002/14651858.CD011975. [PubMed: 26617074]. [PubMed Central: PMC6465076].

5. WulffCB, Gerds TA, Rode L, Ekelund CK, Petersen OB, Tabor A, et al. Risk of fetal loss associated with invasive testing following combined firsttrimester screening for Down syndrome: a national cohort of 147,987 singleton pregnancies. Ultrasound Obstet Gynecol. 2016;47(1):38-44 doi: 10.1002/uog.15820. [PubMed: 26581188].

6. Kazemi M, Salehi M, Kheirollahi M. Down syndrome: Current status, challenges and future perspectives. Int J Mol Cell Med.2016;5(3):125-33. [PubMed: 27942498]. [PubMed Central: PMC5125364]. 